\title{
FACTORES DE RIESGO ERGONÓMICO ASOCIADOS A LA PRODUCTIVIDAD EN EL ÁREA DE TORNO EN UNA EMPRESA DEL SECTOR METALMECÁNICO
}

\author{
ERGONOMIC RISK FACTORS ASSOCIATED WITH PRODUCTIVITY IN THE LATHE AREA \\ IN A METAL-MECHANIC COMPANY
}

Alba Lucía Moreno Burgos ${ }^{1}$

\begin{abstract}
Resumen: La operación de tornos en la industria conlleva a la exposición a diferentes factores de riesgo ergonómicos, como esfuerzos físicos, movimientos repetitivos, deficiencias en el ambiente físico de trabajo, riesgos de carga mental y organización del trabajo. Esto puede contribuir a la aparición de lesiones laborales y, a la vez, generan un impacto negativo en la organización como ausentismo, retraso en procesos o disminución de la productividad. El presente estudio evalúa diferentes factores de riesgo, establece conclusiones y plantea a la empresa propuestas de mejora que no solo contribuyan a elevar la productividad, sino también a la prevención de riesgos laborales y a la salud de los trabajadores. Se emplearon diferentes métodos, entre ellos: el análisis de reprocesos, factores de riesgo ambientales como ruido, estrés térmico, iluminación, estado de las máquinas, evaluación de puestos de trabajo, análisis de sintomatología dolorosa, estimación de la carga de trabajo y evaluación global de puestos de trabajo bajo el método LEST. Como factores críticos, se destacan el estrés térmico ya que, el área presenta una temperatura por encima de los máximos permisibles. En cuanto a la evaluación del puesto de trabajo, las tareas de lijado manual, montar, desmontar piezas y mover la posición de una herramienta, son actividades con mayor riesgo, porque requieren fuerza en los movimientos y posiciones forzadas, que requieren intervención. Para intervenir el factor de riesgo se plantean controles de ingeniería y controles administrativos.
\end{abstract}

Palabras clave: Ergonomía, productividad, factores de riesgo, intervención.

Abstract: The operation of lathes in the industry leads to different ergonomic risk factors, such as physical efforts, repetitive movements, deficiencies in the physical work environment, mental health risks and work organization. This can contribute to work injuries and, at the same time, generate a negative impact on the organization like absenteeism, delay in processes or decrease in productivity. The current applied study evaluates different risk factors, establishes conclusions and proposes improvements to the company that not only contribute to increase productivity, but to the prevention of labor risks and the workers' health. Different methods were used, among them: analysis of reprocessing, environmental risk factors such as noise, thermal stress, lighting, machine condition, evaluation of workplaces, analysis of painful symptoms, estimation of workload and overall evaluation of workplaces under the LEST method. As far as the evaluation of the work place is concerned, the tasks of manual sanding, assembling, dismantling pieces and moving the position of a tool are activities with higher risk because they require strength in the movements and forced positions that

1nvestigadora independiente. Armenia, Colombia. Correo electrónico: albalucia911@gmail.com.

Orcid: https://orcid.org/oooo-0001-6414-8459 
need intervention. To intervene the risk factor, engineering and administrative controls are proposed.

Keywords: Ergonomics, productivity, risk factors, intervention.

Recepción: 02.10.2020 / Revisión: 05.10.2020 / Aceptación: 30.11 .2020

\section{Introducción}

Según el segundo informe de enfermedad profesional en Colombia, 2003 - 2005 (Tafur, 2007), la primera causa de enfermedad profesional registrada en las Administradoras de Riesgos Laborales (ARL) privadas durante el año 2005 fue el síndrome de conducto carpiano (21\%), en segundo lugar la hipoacusia neurosensorial (6\%), en tercer lugar las lesiones osteomusculares y ligamentosas (5\%), en cuarto lugar las sinovitis y tenosinovitis de las manos (3\%), seguidas de la lumbalgia y el síndrome de manguito rotador, cada una con el $2 \%$ de los diagnósticos. Estos diagnósticos son el 40\% de los registrados ese año. Al agrupar las enfermedades por sistemas, los desórdenes musculoesqueléticos son la mayor causa de morbilidad profesional (43\%), mientras que el síndrome del conducto carpiano ocasionó mayor número de días de incapacidad temporal, seguido por las hernias de disco intervertebral y el síndrome de manguito rotador. En consecuencia, estas patologías causan un impacto significativo sobre los trabajadores y sus familias, la productividad de las empresas y sobre el Sistema General de Riesgos Profesionales (SGRP) en términos de costos. Para el 2004, las Administradoras de Riesgos Profesionales (ARP) privadas pagaron 31 indemnizaciones por incapacidad permanente parcial (IPP) generadas por estas enfermedades profesionales. El mayor número de indemnizaciones fue ocasionado por el síndrome del conducto carpiano, seguido de la patología lumbar y la hipoacusia neurosensorial. Estas tres patologías generaron el 90\% de las indemnizaciones por IPP. Durante el año 2005 la industria manufacturera presentó el mayor número de enfermedades profesionales, un $27 \%$ de los sectores reportados.

Según Gaither y Frazier, citados en López et al. (2012), tres factores hacen más productivos a los empleados: 1) el desempeño del puesto del empleado; 2) la tecnología, las máquinas, las herramientas y los métodos de trabajo que apoyan y ayudan su trabajo, y 3) la calidad del producto. Así, la reducción de los defectos, el desperdicio y el trabajo incrementan directamente la productividad de todos los factores de producción. La organización Internacional del Trabajo (OIT) define la productividad como la relación entre la producción obtenida y los recursos utilizados para obtenerla (Prokopenko, 1989, p.3). También puede definirse aritméticamente a través de la formula; productividad = OUTPUT/ INPUT, donde OUTPUT corresponde al valor de los bienes o servicios producidos, y el INPUT, a los recursos invertidos (Vallejo, 2000).

Un sistema de producción puede no producir los resultados esperados como consecuencia de un mal diseño inicial del sistema o por problemas de planeación, análisis y control una vez que el sistema ya se ha implementado (distribución de la planta, manejo de materiales, adquisición de equipo capital para la producción, métodos o tiempo para hacer el trabajo). Con frecuencia estos cinco aspectos se diseñan y desarrollan sin tomar en cuenta al 
trabajador que va a desempeñar la actividad. Las habilidades físicas y mentales del ser humano tienen un límite, las cuales pueden reducirse con los problemas en el diseño del puesto de trabajo.

Las habilidades físicas del trabajador que utiliza para producir (performance físico) pueden estar influenciadas por diferentes factores como los somáticos (sexo, edad, dimensiones corporales y el estado de salud), los psicológicos (motivación, adaptación), el entrenamiento, naturaleza del ejercicio (intensidad, duración, técnica, posición y ritmo) y el medio ambiente (altitud, calor, frío, ruido, contaminación ambiental). En cuanto a las habilidades mentales que requiere el trabajador para desarrollar una tarea (performance mental) son afectadas por factores como los personales (preparación técnica, motivación, estrategias de trabajo), administrativos-organizacionales, uso de tecnología, medio ambiente físico (diseño del lugar de trabajo, ruido, químicos, iluminación y temperatura).

Según Vallejo (2000), cuando el límite de las habilidades físicas se sobrepasa, el trabajador puede desarrollar diferentes problemas que van desde la fatiga hasta la aparición de lesiones musculoesqueléticas irreversibles. Al extralimitar las habilidades mentales pueden darse errores en el desarrollo de la tarea o hasta la aparición de neurosis laboral. Aunque es cierto que los problemas más graves (lesiones irreversibles o neurosis laboral) tardan mucho tiempo en aparecer, también en cierto que un trabajador fatigado física y mentalmente comete errores y disminuye su nivel de producción aun desde el primer día en el puesto de trabajo.

Este estudio tiene como objetivo analizar factores de riesgo organizacionales, diseño de puestos de trabajo, carga física, mental y aspectos del ambiente de trabajo que pueden estar relacionados con la productividad del área de torno en una empresa del sector metalmecánico en la ciudad de Cali, Colombia. Asimismo, evaluar los riesgos ergonómicos presentes en el área de torno del subsistema de producción y establecer las medidas de intervención de acuerdo con los resultados obtenidos.

\section{Materiales y métodos}

La presente investigación es de tipo descriptivo. Se basó en la observación de actividades para conocer el subsistema de producción de la empresa, que tiene un total de cinco operarios, quienes realizan el mecanizado de piezas en cuatro tornos convencionales. Además, en el análisis de reprocesos o devolución de piezas en un periodo de tiempo dado, de factores de riesgo ambientales como el ruido, el estrés térmico, estado de las máquinas, evaluación de puestos de trabajo (diseño y posturas) y carga mental; la observación directa de la realización de la labor para conocer el subsistema; uso de entrevistas al personal; análisis de registros como matriz de peligros y riesgos, caracterización demográfica, ausentismo laboral. Dentro de las técnicas aplicadas están la medición de frecuencia cardiaca para estimar la carga de trabajo, determinación de posturas y movimientos de trabajo, cálculo del índice de masa corporal, mediciones de iluminación, ruido y estrés térmico e inspecciones de seguridad para determinar factores de riesgo comunes que permitan a la empresa la implementación de estrategias de intervención acorde con los resultados. 


\section{Criterios de referencia para la evaluación de los factores de riesgo}

Índice de masa corporal. Según lo indicado por la clasificación de la Organización Mundial de la Salud (De Onis, 2015).

Iluminación. Reglamento Técnico de Iluminación y Alumbrado Público "RETILAP” expedido por la Resolución No 18 1331. (AGO 06 2009), Tabla 404.1, modificado y aclarado mediante las resoluciones 18 o540 de marzo 30 de 2010, 181568 de septiembre 1 de 2010, 182544 de diciembre 29 de 2010, 180173 de febrero 14 de 2011, y 91872 de diciembre 28 de 2012 (Ministerio de Minas y Energía, 2009).

Ruido. Resolución 1972 de 1990, Artículo 1, p.2 (Ministerios de Trabajo y Seguridad Social y de Salud, 1990) y, la Resolución No. 2844 DE 2007 (Ministerio de Protección Social, 2007).

Temperatura. Para realizar la evaluación del ambiente térmico se tuvo en cuenta el índice de WBGT calculado con temperatura húmeda, temperatura de globo y temperatura seca y la exposición promedio ocupacional de acuerdo con lo establecido en el parágrafo del artículo 64 de la Resolución 2400 (Ministerio de Trabajo y Seguridad Social, 1979). Como referencia se toman los Valores Límites Permisibles (TLVs) 2014 de ACGIH, aceptados en Colombia por la normatividad legal (ARSEG, 2012). Fórmula de cálculo empleada: IWBGT = 0,7Th + 0,3 $\mathrm{Tg}$ (sin exposición directa al sol).

Espacios de trabajo y circulación. Se tomaron como referencia los parámetros establecidos en la Resolución 2400 de 1979 (Artículo 12) y la NTC 4140 de 2005 (numeral 3.1) sobre características generales de edificios, pasillos y corredores (Instituto Colombiano de Normas Técnicas y Certificación [ICONTEC], 2005). Se establece el ancho mínimo de un pasillo para acceso de público de 1,20 m de la Resolución 2400 el artículo 9; la superficie de pavimento por trabajador no será menor de $2 \mathrm{~m} 2$ y el artículo 12 (parágrafo 1), la distancia entre máquinas, aparatos, equipos y otros, no será inferior a o,80 m (ARSEG, 2012).

Altura de la superficie de trabajo. De o a $10 \mathrm{~cm}$ por encima del codo, lo cual sirve de soporte reduciendo las cargas estáticas en los hombros.

Ángulos de movilidad articular miembros superiores. Según se detalla en el anexo 1.

Estimación de la carga de trabajo. Se empleó la escala de Christensen modificada para estimar la carga de trabajo con base en mediciones de frecuencia cardiaca (Christensen, citado en Castillo \& Cubillos, 2014).

Evaluación global del puesto de trabajo. Aplicación del sistema de evaluación método LEST (Chiner et al., 2004).

\section{Técnicas e instrumentos de evaluación}

1. Revisión documental sobre aspectos organizacionales del trabajo como perfil del cargo, certificados de aptitud laboral y paraclínicos para precisar los requerimientos psíquicosfísicos del cargo y establecer su relación con los hallazgos clínicos.

2. Análisis de reprocesos y devoluciones de las piezas fabricadas o reparadas por operario durante el 2014. Se revisaron los registros de no conformes del año 2014 donde se establece 
el porcentaje de reprocesos internos o devoluciones por el cliente del total de órdenes generadas por operario y el costo del mismo en relación con el salario del trabajador.

3. Comprobación del funcionamiento de los cuatro tornos mediante inspección de seguridad que incluye la verificación del sistema eléctrico, niveles de aceite, partes eléctricas, componentes metálicos, herramientas de corte y aspectos de seguridad y limpieza de las máquinas, aplicando lista de chequeo de verificación de tornos (anexo 2).

4. Estimación de la carga de trabajo mediante mediciones de frecuencia cardiaca (Christensen, citado en Castillo \& Cubillos, 2014), de los operarios durante cuatro horas de la jornada. Se tomó la moda de un periodo de reposo con el operador sentado durante 10 minutos antes de iniciar el trabajo. Se llevó el registro de la frecuencia cardiaca cada media hora, consolidando los resultados por hora e indicando las tareas que el operario estaba realizando en el lapso de tiempo en que se presentó el aumento más significativo de la frecuencia de trabajo. Se empleó un reloj Polar FT60, con su respectiva banda Polar Wearlink.

Tabla 1. Clasificación de la carga de trabajo según frecuencia cardiaca en latidos por minuto.

\begin{tabular}{|c|c|}
\hline Frecuencia cardiaca & Carga fisiológica de trabajo \\
\hline 75 & Muy baja \\
\hline $75-100$ & Baja \\
\hline $115-130$ & Alta \\
\hline $130-150$ & Muy alta \\
\hline Sobre 150 & Extremadamente alta \\
\hline
\end{tabular}

5. Análisis de la actividad. Mediante observación directa de la realización de la labor, medición de la superficie de pavimento por trabajador, la distancia entre máquinas y equipos y el espacio para el tránsito de personal con ayuda de un decámetro de los cuatro puestos de trabajo. También la altura de trabajo de pie, determinación de posturas de trabajo obteniendo registro fotográfico de las posturas más desfavorables de miembros superiores y realizando medición de ángulos de confort mediante el programa Kinovea y aplicación del Cuestionario Nórdico Modificado (Kourinca et al. citados en Cuestionario Nórdico, s.f.).

6. Medición del estrés térmico. Para la cuantificación de las condiciones de estrés térmico en el área de torno se instalaron durante 15 minutos monitores QUESTEMP ${ }^{\circ} 36$ serial TKP 030015, el cual presenta los resultados de temperatura de bulbo seco (Tbs), temperatura de bulbo húmedo (Tbh), temperatura de globo ( $\mathrm{Tg}$ ), WBGT para interiores y WBGT para exteriores, aceptado internacionalmente por entidades como la NIOSH, OSHA y ACGIH, y el medidor de velocidad Quest - 3M serial WPPo50002. Para la evaluación de la carga térmica ambiental se realizó la medición en el torno más distante del área, retirado de la puerta de salida y donde la circulación de aire es más deficiente, ubicando el equipo a la altura del tórax del trabajador esperando que se estabilice (entre 15 y 20 minutos). Las condiciones climáticas del día de medición fueron de día seco y despejado entre las 11 a.m. y la 1 p.m. donde predomina la mayor parte del calor. 
7. Evaluación de iluminación. Niveles de iluminación en los puestos de trabajo, con el trabajador en su posición normal, en el punto donde realiza el esfuerzo, con un luxómetro marca Hagner.

8. Evaluación ambiental de ruido para determinar la exposición ocupacional y el grado de riesgo. Se realizó una sonometría por el tipo de ruido continuo que se presenta en el área. Se empleó un sonómetro Quest Tecnologies modelo VI-400PRO Vibration Monitor Serial: 9268 y un calibrador acústico Quest Tecnologies modelo QC -10 Serial: QE5120158.

9. Aplicación del método LEST, para la evaluación de las condiciones de trabajo de forma global: entorno físico, la carga física, la carga mental, psicosociales y tiempos de trabajo, para determinar si las situaciones son satisfactorias, molestas o nocivas. Se empleó el cuestionario adaptado al Programa e-LEST (Chiner et al., 2004).

\section{Resultados y discusión}

\section{Análisis de registros}

En el subsistema de producción no se realizan labores en serie. Estas están distribuidas así: torneros 1 y 3 mecanizan piezas pequeñas y cuando se requieren por volumen; torneros 2, 4 y 5 piezas medianas y grandes. En la matriz de peligros y riesgos se identifican como riesgos prioritarios el mecánico, el biomecánico y el físico (exposición a ruido y disconfort con la temperatura ambiental), estos tres últimos relacionados con el objeto del estudio. En la caracterización sociodemográfica se encontró que los cinco operarios son hombres, con un salario básico que oscila entre uno y dos salarios mínimos mensuales vigentes, el 60\% son mayores de 50 años, el 80\% tienen relaciones de tipo estable, el 60\% cuenta con un nivel de escolaridad igual o superior al bachillerato, requisito mínimo exigido por el perfil del cargo, el 80\% lleva 3 años o más laborando en la empresa, y se labora en promedio 48 horas semanales (con dos descansos de 15 minutos y pausas activas de 3 minutos en la mañana y en la tarde). En cuanto al ausentismo laboral 2014, se presentaron 9 días de incapacidad, correspondientes a cuatro eventos por enfermedad general y uno por accidente de trabajo, con 7 y 2 días de incapacidad respectivamente, sin ninguna relación con el objeto del estudio.

\section{Revisión documental sobre aspectos organizacionales del trabajo como perfil del cargo, certificados de aptitud laboral}

Solo uno de los trabajadores no cumple con el nivel de escolaridad requerido, sin embargo, cuenta con la formación y la experiencia mínima requerida para el desempeño del cargo de 3 años. En cuanto a los requerimientos físicos del cargo, las audiometrías indican una audición normal bilateral en cuatro de los cinco operarios, en el otro, baja auditiva para frecuencias desde 3.000 $\mathrm{Hz}$ bilateral de grado moderado. Las optometrías indican que dos operarios requieren el uso de lentes formulados para corregir vicios de refracción, tres de ellos usan gafas de seguridad con adaptación de lentes formulados, y los demás requieren valoración para cambio de fórmula. En cuanto a los certificados de aptitud para el cargo de 2011, en el aspecto osteomuscular, hay un operario con alteraciones y uno sintomático en miembros 
superiores, y dos operarios con alteraciones en el sistema cardiovascular. Al calcular el índice de masa corporal (IMC) se encontró que un operario tiene obesidad tipo I y otro tiene sobrepeso grado I. De acuerdo a los certificados médicos de aptitud laboral de 2011, hay diagnóstico de síndrome de manguito rotador derecho en el operario 1 y referencia de dolor en el codo derecho del operario 3.

\section{Análisis de reprocesos y devoluciones de las piezas fabricadas o reparadas por operario durante el 2014}

Las devoluciones de las piezas maquinadas por parte de los clientes costaron en el 2014 $\$ 2.469 .783$ equivalentes al 3\% del valor pagado a los operarios como honorarios. De las 2.902 órdenes generadas, 70 (2\%) fueron no conformes y 9 (13\%), fueron piezas devueltas por el cliente, porque tuvieron dimensiones diferentes a las solicitadas. o Los trabajadores argumentan que la diferencia puede deberse a i. la pérdida de la concentración, ii. al uso de herramientas defectuosas y, en último lugar y, iii. a la falta de mantenimiento preventivo de los tornos.

\section{Comprobación del funcionamiento de los tornos mediante inspección de seguridad}

Uno de los tornos presenta fallas en el freno del pie, es operado por los trabajadores 2 y 5 . El operario 5 presentó en el 2014 el mayor número de reprocesos o devoluciones por parte del cliente de las piezas mecanizadas, y el operario 2 está en tercer lugar.

\section{Estimación de la carga de trabajo}

Se evaluaron tres de los cinco operarios. Se obtuvo que la carga fisiológica del trabajo es baja ya que en todos los casos la frecuencia cardiaca se mantuvo entre 75 y 100 latidos por minuto y no se supera en ningún momento el $40 \%$ de la carga cardiovascular para considerar el trabajo como pesado. Las personas más altas tienen las pulsaciones más bajas. En cuanto a la realización de la labor es de anotar que en dos de los tres casos evaluados el aumento de la frecuencia cardiaca se debió a la variación en las tareas. 
Tabla 2. Carga fisiológica del trabajo mediante mediciones de frecuencia cardiaca.

\begin{tabular}{|c|c|c|c|c|c|c|c|c|c|c|c|c|}
\hline VARIABLES & \multicolumn{4}{|c|}{ OPERARIO 1} & \multicolumn{4}{|c|}{ OPERARIO 2} & \multicolumn{4}{|c|}{ OPERARIO 5} \\
\hline Edad & \multicolumn{4}{|c|}{51} & \multicolumn{4}{|c|}{60} & \multicolumn{4}{|c|}{49} \\
\hline $\begin{array}{c}\text { FC reposo } \\
\text { lat } / \mathrm{min}\end{array}$ & \multicolumn{4}{|c|}{63} & \multicolumn{4}{|c|}{76} & \multicolumn{4}{|c|}{84} \\
\hline FC máxima & \multicolumn{4}{|c|}{169} & \multicolumn{4}{|c|}{160} & \multicolumn{4}{|c|}{171} \\
\hline \multirow[t]{2}{*}{ FC trabajo } & $\begin{array}{c}1^{\mathrm{a}} \\
\text { hora }\end{array}$ & $\begin{array}{c}2^{\mathrm{a}} \\
\text { hora }\end{array}$ & $\begin{array}{c}3^{a} \\
\text { hora }\end{array}$ & $\begin{array}{c}4^{\mathrm{a}} \\
\text { hora }\end{array}$ & $\begin{array}{c}1^{\mathrm{a}} \\
\text { hora }\end{array}$ & $\begin{array}{c}2^{\mathrm{a}} \\
\text { hora }\end{array}$ & $\begin{array}{c}3^{a} \\
\text { hora }\end{array}$ & $\begin{array}{c}4^{\mathrm{a}} \\
\text { hora }\end{array}$ & $\begin{array}{c}1^{\mathrm{a}} \\
\text { hora }\end{array}$ & $\begin{array}{c}2^{\mathrm{a}} \\
\text { hora }\end{array}$ & $\begin{array}{c}3^{a} \\
\text { hora }\end{array}$ & $\begin{array}{c}4^{a} \\
\text { hora }\end{array}$ \\
\hline & 71 & 75 & 76 & 77 & 77 & 77 & 82 & 87 & 88 & 91 & 84 & 81 \\
\hline $\begin{array}{l}\text { FC trabajo } \\
\text { promedio }\end{array}$ & \multicolumn{4}{|c|}{75} & \multicolumn{4}{|c|}{81} & \multicolumn{4}{|c|}{86} \\
\hline $\mathrm{CC} \%$ & 7,5 & 11,3 & 12,3 & 13,2 & 1,2 & 1,2 & 7,1 & 13 & 4,6 & 8,0 & 0 & 1,1 \\
\hline CC\% Promedio & \multicolumn{4}{|c|}{11,8} & \multicolumn{4}{|c|}{5,6} & \multicolumn{4}{|c|}{3,4} \\
\hline
\end{tabular}

\section{Análisis de la actividad}

Un ciclo de trabajo puede tener una duración de 15 minutos a 4 horas dependiendo del tamaño y la complejidad de la pieza a mecanizar. Las tareas realizadas por los operarios de torno durante una jornada de trabajo se describen en el anexo 3. La tarea de elaboración o reparación de piezas en torno abarca el mayor tiempo de la jornada, por lo cual fue analizada. Las actividades se realizan en bípedo, por lo cual los miembros inferiores soportan el peso corporal; se desarrollan en un plano medio de trabajo (por debajo de los hombros). Requieren de actividad bimanual (dos manos) con mayor protagonismo de miembro superior derecho, se debe ejercer fuerza de presión y agarre. El hombro trabaja entre el rango funcional $90^{\circ}$, ejerce fuerza isométrica pues está en el aire sin ningún tipo de apoyo, esto favorece la aparición de fatiga de forma rápida. El codo adopta posición entre $60 \mathrm{y} 100^{\circ} \mathrm{de}$ flexión, la muñeca adopta diversas posiciones entre flexo extensión, desviaciones radial y ulnar de acuerdo a la ubicación de la manivela. Se debe ejercer diferentes tipos de agarre y actividades de motricidad fina. Todos los mandos de las máquinas se encuentran situados dentro de la esfera fisiológica o de alcance de las dos manos (anexo 4).

En cuanto a la distancia entre máquinas, en 3 tornos es inferior a $80 \mathrm{~cm}$, lo cual puede generar golpes, dificultar el acceso de los trabajadores a sus puestos de trabajo y la evacuación en caso de emergencia, y disconfort con la temperatura del ambiente al no permitir la fácil circulación del aire, que puede llevar a pérdidas de la concentración y los consecuentes accidentes de trabajo o daño de las piezas trabajadas, así como de las herramientas utilizadas. Los pasillos principales tienen un ancho superior a $120 \mathrm{~cm}$, el indicado para corredores de uso público.

El cuestionario nórdico modificado se aplicó a cuatro de los cinco operarios, un operario se negó a diligenciarlo. Todos refieren sintomatología dolorosa en miembros superiores en los últimos doce meses en hombro derecho, ambas muñecas, codo derecho y 
hombro izquierdo. En el análisis de las tareas realizadas, el lijado manual, montar y desmontar la pieza y mover la posición de una herramienta, son las operaciones que representan mayor riesgo para la aparición de desórdenes musculoesqueléticos de miembros superiores ya que requieren fuerza en los movimientos y posiciones de extensión o rotación forzadas de la muñeca o la mano.

\section{Medición de estrés térmico}

Los operarios usan camisas manga larga y corta de dril color azul oscuro, no hay evidencia de sistemas de ventilación localizada. Hay calentamiento por tejas asbesto cemento. La puerta de acceso a la planta se encuentra todo el tiempo abierta para que ingresen corrientes de aire al área. No hay un punto de hidratación, solo un lavadero donde pueden tomar agua, que no es la estructura adecuada. El área de torno presenta niveles de estrés térmico (WBGT) por encima de los valores máximos permisibles, que para un trabajo moderado (con los dos brazos) se estima en $28{ }^{\circ} \mathrm{C}$, y la medición arroja $30,5^{\circ} \mathrm{C}$. Gran parte de los niveles de calor se debe a la poca circulación de las corrientes de aire $(0,09 \mathrm{~m} / \mathrm{s})$, este valor según los parámetros establecidos en la norma debería estar entre 1,3 y 1,5 m/s. También al calor radiante producido por el material del techo, el color oscuro de la ropa, de las superficies de trabajo (base en madera que permanece engrasada) y las máquinas presentes en el área. Dado que las personas obesas o mayores de 50 años tienen mayores riesgos de trabajar en puestos de calor debido a la deficiencia en los sistemas pulmonar y cardiovascular, es importante destacar que tres de los cinco operarios son mayores de 50 años, dos son hipertensos (controlados), y uno tiene un IMC de 30,9 lo cual indica obesidad tipo I, razón por la cual es importante establecer medidas de intervención para el control de los riesgos descritos.

Tabla 3. Resultados de medición de estrés térmico.

\begin{tabular}{|c|c|c|c|c|c|c|c|c|c|c|}
\hline $\begin{array}{c}\text { Tbh } \\
\left({ }^{\circ} \mathrm{C}\right) \\
\text { Media }\end{array}$ & $\begin{array}{c}\text { Tbs } \\
\left({ }^{\circ} \mathrm{C}\right) \\
\text { Media }\end{array}$ & $\begin{array}{c}\mathrm{Tg}\left({ }^{\circ} \mathrm{C}\right) \\
\text { Media }\end{array}$ & $\begin{array}{c}\text { WBG } \\
\mathrm{T} \\
\left({ }^{\circ} \mathrm{C}\right)\end{array}$ & $\begin{array}{c}\text { Velo- } \\
\text { cidad } \\
\text { aire } \\
\mathrm{m} / \mathrm{s}\end{array}$ & $\begin{array}{c}\text { Humedad } \\
\text { Relativa }\end{array}$ & $\begin{array}{c}\text { WBGT } \\
\text { norma }\end{array}$ & $\begin{array}{c}\text { Nivel } \\
\text { de } \\
\text { acción }\end{array}$ & $\begin{array}{c}\text { Régimen } \\
\text { Trabajo } \\
\text { Descanso }\end{array}$ & $\begin{array}{c}\text { Carga } \\
\text { Metabólica }\end{array}$ & Riesgo \\
\hline 27.3 & 34.1 & 36.8 & $\begin{array}{c}30.5^{\circ} \\
\mathrm{C}\end{array}$ & $\begin{array}{c}0.09 \\
\mathrm{~m} / \mathrm{s}\end{array}$ & $\mathbf{6 0 \%}$ & 28.0 & 25.0 & $\mathbf{7 5 - 1 0 0}$ & Moderado & Alto \\
\hline
\end{tabular}

\section{Medición de iluminación}

Los puestos de trabajo presentan niveles adecuados de iluminación. No obstante, es importante considerar que el trabajo de mecanizado de piezas en torno es una labor que requiere precisión. La causa de los reprocesos y devoluciones que se dieron en 2014 corresponde a piezas fabricadas o reparadas con medidas diferentes a las solicitadas en los planos, que pueden asociarse a factores físicos de los operarios y ambientales de los puestos de trabajo, dado que los operarios 1 y 5 requieren valoración por optometría para cambio de fórmula, a su vez, son los operarios que más reprocesos y devoluciones de piezas por parte de los clientes tuvieron en 2014. El torno número 4 (operado por el trabajador 1) presenta un tubo de luminaria fundido y las luminarias presentan falta de limpieza. 
Tabla 4. Niveles de iluminación medidos comparados con los niveles del RETILAP.

\begin{tabular}{|c|c|c|c|c|}
\hline No. & PUESTO & $\begin{array}{l}\text { NIVEL } \\
\text { PROMEDIO } \\
\text { DEL } \\
\text { PUESTO } \\
\text { (LUX) }\end{array}$ & $\begin{array}{l}\text { NIVEL } \\
\text { RECOMENDADO } \\
\text { (LUX) }\end{array}$ & TIPO RECINTO Y ACTIVIDAD \\
\hline \multicolumn{5}{|c|}{ ¿NIVELES DENTRO DE LO RECOMMENDADO 口SOBRE EXCESO } \\
\hline 1 & Torno \#1 & 1050 & \multirow{4}{*}{$500-750-1000$} & \multirow{4}{*}{$\begin{array}{l}\text { Taller de mecánica y ajuste } \\
\text { maquinado y trabajo fino en } \\
\text { banco, maquinas automáticas } \\
\text { finas, inspección y ensayos }\end{array}$} \\
\hline 2 & Torno $\| 2$ & 809 & & \\
\hline 3 & Torno \#3 & 575 & & \\
\hline 4 & Torno \#4 4 & 681 & & \\
\hline
\end{tabular}

\section{Evaluación ambiental del ruido}

El mayor ruido registrado corresponde al uso de pulidoras DEWALT durante el pulido con disco-lija y disco-piedra respectivamente, con niveles de presión sonora elevados (96,1 y 107,5 dBA) y tiempos de exposición cortos. Según el análisis de protección auditiva realizado para el nivel de ruido más alto (pulidora DEWALT industrial con disco de piedra), el protector auditivo tipo anatómico suministrado por la empresa cumple con los niveles de atenuación requeridos para los niveles de ruido registrados durante el monitoreo. Los tornos presentan niveles de generación de ruido inferiores al valor límite permisible, sin embargo, se debe tener precaución con la exposición frecuente, pues el ruido se presenta en el orden de nivel de acción (8o dBA).

Tabla 5. Evaluación ambiental del ruido.

\begin{tabular}{|c|c|c|c|c|c|c|c|c|c|c|}
\hline \multirow[t]{2}{*}{ PUNTO EVALUADO } & \multirow{2}{*}{$\begin{array}{l}\text { NPS } \\
\mathrm{Db}(\mathrm{A})\end{array}$} & \multirow{2}{*}{$\begin{array}{c}\text { NPS } \\
\text { Db(Lin) }\end{array}$} & \multicolumn{8}{|c|}{ ANÁLISIS DE FRECUENCIA (Db(Lin)) } \\
\hline & & & 63 & 125 & 250 & 500 & $1 \mathrm{k}$ & $2 k$ & $4 k$ & $8 k$ \\
\hline $\begin{array}{c}\text { Torno Pinacho Latino } \\
\text { Mod L- } 1 / 225\end{array}$ & 80.0 & 84.3 & 72.2 & 68.7 & 72.7 & 75.7 & 77.0 & 73.6 & 67.5 & 63.4 \\
\hline Maquina $\mathrm{CNC}$ & 75.1 & 81.6 & 76.1 & 76.5 & 71.6 & 72.6 & 72.9 & 67.0 & 63.9 & 60.6 \\
\hline Taladro de Árbol & 83.2 & 86.7 & 72.4 & 77.5 & 72.3 & 77.8 & 78.2 & 78.6 & 79.5 & 72.1 \\
\hline $\begin{array}{c}\text { Pulidora DEWALT } \\
\text { Industrial }\end{array}$ & 96.1 & 97.5 & 70.5 & 73.4 & 83.2 & 79.1 & 88.7 & 90.4 & 93.7 & 87.3 \\
\hline $\begin{array}{c}\text { Pulidora DEWALT } \\
\text { Industrial }\end{array}$ & 107.5 & 110.8 & 45.4 & 60.2 & 68.8 & 86.0 & 98.3 & 101.9 & 102.5 & 100.3 \\
\hline
\end{tabular}
RESULTADOS BASADOS EN GUIA - GATISO
Analisis para una tasa de intercambio de 3 dB $=<81,9 \mathrm{~dB}$

$82-99,9 \mathrm{~dB}$

- $>100 \mathrm{~dB}$
RESULTADOS BASADOS EN LA RESOLUCION 1792 Analisis para una tasa de intercambio de 6 dB

$=<79,9 \mathrm{~dB}$

$80,0-84,9 \mathrm{~dB}$

= $>85,0 \mathrm{~dB}$ 


\section{Aplicación del método LEST}

Para su aplicación se tuvieron en cuenta los resultados de las mediciones de estrés térmico, de iluminación y presión sonora, así como la opinión de los trabajadores respecto a las labores que realizan. La dimensión con mayor puntuación de 10 (nocividad) corresponde al entorno físico por el nivel de estrés térmico (WBGT) (con valores máximos permisibles) y el ruido de nivel de acción (8o dBA) y exposición continua. Las dimensiones tiempo de trabajo, carga mental y aspectos psicosociales obtuvieron una puntuación de 6-7 (molestias medias, existe riesgo de fatiga). Los trabajadores deben permanecer en su puesto de trabajo hasta finalizar la jornada laboral, aunque hayan terminado sus labores, no pueden fijar el momento y duración de las pausas. La carga mental por el nivel de atención requerido para la tarea es elevada; se requiere precisión pues por las revoluciones que manejan los tornos hay una mayor probabilidad de sufrir accidentes de trabajo con consecuencias graves como la amputación de dedos. Esto reviste de una mayor complejidad a la tarea para evitar reprocesos y devoluciones de las piezas mecanizadas. En relación con los aspectos psicosociales (variable relación con el mando), la obtención de resultados de producción del área depende del desempeño eficiente de otros puestos de trabajo pertenecientes al subsistema de gestión de los recursos (compras para el suministro de materiales de trabajo) y al subsistema de gestión comercial (diseñadores para el suministro de planos con las especificaciones de diseño, reparación o fabricación de piezas).

\section{Conclusiones}

Si bien es cierto en el año 2014 no hubo reportes de ausentismo por eventos relacionados con el sistema osteomuscular, en los certificados médicos de aptitud laboral de 2011 hay diagnóstico de síndrome de manguito rotador derecho y dolor en el codo derecho en dos operarios. La sintomatología dolorosa que presentan los operarios por las tareas realizadas de lijado manual, montaje y desmonte de piezas, así como mover de posición una herramienta, representan mayor riesgo para la aparición de desórdenes musculoesqueléticos de miembros superiores. Esto podría ocasionar una enfermedad de tipo laboral con costos directos (pago por un trabajo no realizado; tratamiento médico e indemnización; la reparación o la sustitución de máquinas y equipos dañados; la disminución o la interrupción temporal de la producción; el aumento de los gastos en formación y administración; la posible disminución de la calidad del trabajo) e indirectos (sustitución del trabajador enfermo; formación de un nuevo trabajador y darle tiempo para que se acostumbre al puesto de trabajo), afectando la productividad de la empresa.

$\mathrm{Al}$ realizar las actividades, la mayor parte del tiempo en bípedo, los miembros inferiores soportan el peso corporal, lo cual podría ocasionar lumbalgia, dolor o hinchazón en pies y piernas, fascitis plantar, restricción del flujo sanguíneo, venas varicosas, incremento de cambios óseo degenerativos. La carga fisiológica del trabajo es baja y no se supera el $40 \%$ de la carga cardiovascular para considerar el trabajo como pesado.

La condición más crítica encontrada es el área de torno, que presenta niveles de estrés térmico (WBGT) por encima de los valores máximos permisibles. Los efectos psicológicos 
que el calor produce en las personas se relacionan con su eficiencia para desarrollar funciones mentales y para rendir en trabajos físicos. Además, se aumentan la percepción de incomodidad e insatisfacción, la irritabilidad, disminución del estado de alerta y de concentración, las decisiones erróneas, el sueño y la fatiga, más si se tiene en cuenta el estado de salud de algunos operarios (diagnóstico de obesidad, sobrepeso e hipertensión).

Como causas de devolución de las piezas elaboradas, para todos los operarios predominó que estas tuvieron dimensiones diferentes a las solicitadas. Los trabajadores infieren que puede deberse principalmente a pérdida de la concentración, seguido del uso de herramientas defectuosas y, en último lugar, a la falta de mantenimiento preventivo de los tornos. Se puede asociar las condiciones de las luminarias sucias y en mal estado con la disminución de la eficiencia de los trabajadores, ya que una visión y una buena iluminación mejoran el rendimiento. Los operarios que requieren valoración por optometría para cambio de fórmula, a su vez se encuentran dentro de los que más reprocesos y devoluciones de piezas por parte de los clientes tienen. Atender estos aspectos puede contribuir a la reducción de errores y reprocesos, aumenta la seguridad al reducir las tasas de accidentalidad y el ausentismo.

Los tornos presentan niveles de generación de ruido inferiores al valor límite permisible. Sin embargo, se considera tener precaución en la exposición frecuente, pues el ruido se presenta en el orden de nivel de acción (80 dBA). Tal ruido puede generar en los trabajadores no solo hipoacusia sensorial o disminución del nivel de audición, dolor de cabeza y cansancio, sino además pérdida de la concentración, estrés, irritabilidad, conflictos o problemas de actitud, incumplimiento de metas, entre otros.

La empresa no cuenta con programa de mantenimiento preventivo de máquinas, equipos y herramientas. Se realiza únicamente mantenimiento correctivo, lo cual puede estar incidiendo en la calidad de las piezas, diseñadas, reparadas o fabricadas principalmente del torno 2 que presenta fallas en el freno de pie.

\section{Recomendaciones}

- Realizar el análisis costo-beneficio de las intervenciones ergonómicas planteadas en este informe, el cual incluya la determinación de costos, determinación de beneficios y el análisis costo-beneficio de las mismas

- Efectuar valoración médica por EPS (Entidad Promotora de Salud) para establecer diagnóstico y tratamiento de la sintomatología dolorosa en miembros superiores, así como de obesidad tipo I, y control por optometría para formulación de lentes para corregir vicios de refracción.

- Aplicar las baterías de riesgo psicosocial de acuerdo con los lineamientos establecidos en la Resolución 2646 de 2008, por la cual se establecen disposiciones y se definen responsabilidades para la identificación, evaluación, prevención, intervención y monitoreo permanente de la exposición a factores de riesgo psicosocial en el trabajo, y para la determinación del origen de las patologías causadas por el estrés ocupacional.

- Realizar un programa de higiene postural, dirigido a todos los trabajadores con el fin de 
prevenir la aparición temprana de la fatiga muscular y lesiones osteomusculares a largo plazo, control y seguimiento de los casos diagnosticados, así como la medición de indicadores de gestión para determinar la eficiencia del mismo.

- Ampliar el espacio de separación entre máquinas y equipos mediante el retiro del área de torno en desuso y la reorganización de los tornos en uso, conservando espacios mínimos de $80 \mathrm{~cm}$ entre máquinas.

- Diseñar e implementar un programa de mantenimiento preventivo y predictivo a los tornos de la empresa, de acuerdo con las especificaciones del fabricante, como una medida de intervención para reducir el tiempo de exposición al riesgo ya que el ajuste adecuado del torno mejora su eficiencia como lo sugiere Campaña (2013).

- Implementar un plan de aseo a las respectivas luminarias y adecuación de altura, es decir unificar las luminarias de los tornos similar a la del torno 4 con una altura de 40 a 45 pulgadas, para mejorar la incidencia sobre los planos de trabajo. Adicionalmente, incorporar a los balastos la pestaña de incidencia hallada en el torno 4, para que la luz no se disperse, y reemplazar tubo de luminaria fundido en el torno 4.

- Dotar a los puestos de trabajo de apoya pies para reducir la compresión intradiscal, de una altura 10 a $20 \mathrm{~cm}$, distancia desde el borde anterior de la superficie para postura bípeda de 15 a $23 \mathrm{~cm}$, como lo sugieren Panero y Zelkin, citados en Rueda \& Zambrano (2013), para descansar alternadamente los pies.

- Diseñar un esquema de descanso de 60 minutos de trabajo con 5 minutos de descanso, dado que la reducción de la fatiga muscular en un trabajador que labora de pie está demostrado que se logra en los primeros 5 minutos (Konz y Johnson; Van Dieen, citados en Cañas, 2015).

Dado que se presentan altas temperaturas debido a la poca circulación de aire y a la acumulación de aire caliente se recomienda la aplicación de medidas de intervención como:

- Mejorar el ingreso de aire en las zonas evaluadas, realizando la adecuación de sistemas de ventilación mecánica para las entradas de aire, procurando que arrastren el aire limpio a las zonas más calurosas, creando un cierto efecto "ventilación por desplazamiento", ubicando las salidas de aire para lo cual el aire frío (más denso) desplazará el aire caliente, considerando el recorrido esperable del aire en la zona, de modo que, idealmente, la secuencia sea: entrada de aire-trabajador-foco-salida de aire. Prever la reposición del aire extraído y, por último, evitar que el aire extraído vuelva a introducirse en el local (anexo 5).

- Instalar un inyector tipo galpón en la parte frontal, con la finalidad de reducir los niveles de calor a nivel interno de la empresa, que arroje 6250 CFM (pies cúbicos por minuto). Este sistema puede ayudar a dispersar parte del calor radiante. Se debe calcular el caudal de aire necesario teniendo en cuenta las dimensiones de la bodega (anexo 6).

- Tener en cuenta la ventilación por dilución, la cual no puede ser considerada como una solución al problema higiénico planteado, sino como complemento necesario a la extracción localizada. Un sistema de ventilación general puede ser completamente 
mecánico (entradas y salidas mecánicas), natural (entradas y salidas no forzadas) o bien mixto (entrada mecánica y salida natural y viceversa). Además, adecuar o alargar el caballete e instalar otro similar en la parte delantera con la finalidad de mejorar la salida del aire caliente (anexo 7).

- Usar ventiladores para aumentar la velocidad con que circula el aire en el torno 4, lo cual mejorará el intercambio de calor entre la superficie de la piel y el aire (ventilación inducida).

- Cambiar del uniforme de un color oscuro por absorción del calor radiante, por un color claro.

- Adecuar el área de hidratación, la cual debe estar tan cerca como sea posible al lugar de trabajo, sobre todo para mejorar el consumo de agua, pues por la existencia de poca ventilación el índice de sudoración se puede incrementar, al igual que la pérdida de sales minerales.

- Medir de nuevo el estrés térmico, luego de implementar estos cambios, para comprobar que al aumentar velocidad del aire y disminuir el calor radiante disminuye el índice WBGT.

- Implementar un sistema de vigilancia epidemiológica (SVE) de sobrecarga térmica, que incluya criterios de restricción médica para los trabajadores nuevos que laborarán en las áreas calientes; exámenes de ingreso y de retiro, identificación de la población objeto del sistema, valoración periódica de las condiciones del sistema cardiovascular, exámenes médicos periódicos, regular actividades del SVE e indicadores de gestión.

- Realizar actividades educativas participativas, dirigidas al personal expuesto y no expuesto en el cual se informa sobre los métodos más adecuados para prevención, promoción y control de riesgos de temperaturas extremas, incluyendo información relacionada con el sistema de gestión de la seguridad y la salud en el trabajo de la empresa.

- Tener una hidratación adecuada (ingerir agua potable, 1 vaso cada 20 minutos aproximadamente $\mathrm{u}$ otras bebidas hidratantes que contengan sales minerales) durante la jornada laboral, y la ingesta de comidas normales y refrigerios que les suministren suficiente sal y electrolitos para reemplazar los perdidos en el sudor, previa valoración e indicación médica, pues hay que tener en cuenta que los operarios 2 y 4 son hipertensos, siendo este último además diabético.

- Continuar con el uso de protección auditiva, ya que los operarios no se exponen solamente al ruido generado por estos, sino también a las diferentes fuentes de ruido de las áreas de trabajo periféricas. 


\section{Referencias}

ARSEG, (2012). Compendio de normas legales en Colombia ( $\left.2^{\mathrm{a}} \mathrm{Ed}\right)$. Capital Safety.

Campaña, O. (2013). Desarrollo de un sistema de mantenimiento preventivo para las máquinas torno winston - torno pinacho - llenadora de resistencias - fresadora jhonford - swager o reductor, de la empresa COLRES LTDA de interés industrial [tesis de pregrado]. Repositorio Universidad Autónoma de Occidente, Santiago de Cali, Colombia. http://bdigital.uao.edu.co/bitstream/10614/4995/1/TME01378.pdf

Cañas, J. J. (2015). Guía. Buenas prácticas en ergonomía aplicada a la prevención de riesgos laborales de tipo psicosocial. Secretaria de Salud y Medio Ambiente, UGT-CEC. https://www.diba.cat/documents/467843/106587019/GuiaErgonomiaWEB.pdf/8bd4e628ddd2-4513-bf74-9b301b774fbb

Castillo, J. A., \& Cubillos, A. (2014). Uso de la frecuencia del pulso en la estimación de la carga de trabajo. Evaluación de una actividad de movilización de cargas. Revista Ciencias de la Salud, 12 (Especial), 27-43. https://doi.org/10.12804/revsalud12.esp.2014.04

Chiner, M., Diego, J. A, \& Alcaide, J. (2004). Laboratorio de ergonomía (1 a Ed). Alfaomega.

Cuestionario Nórdico (s.f.). Ergonomía en español. https://www.talentpoolconsulting.com/wpcontent/uploads/2014/06/cuestionario-nordico-kuorinka.pdf.

De Onis, M. (2015). Valores de referencia de la Organización Mundial de la Salud. En M.L. Frelut(Ed.), El ebook ECOG'S sobre niños y adolescentes obesos. https://ebook.ecogobesity.eu/es/tablas-crecimiento-composicion-corporal/valores-de-referencia-de-laorganizacion-mundial-de-la-salud/

Instituto Colombiano de Normas Técnicas y Certificación [ICONTEC] (2005). Norma Técnica Colombiana NTC 4140. Accesibilidad de las personas al medio físico. edificios, pasillos y corredores. Características generales. Primera actualización editada. https://www.academia.edu/36690018/NORMA T\%C3\%89CNICA NTC COLOMBIANA 4 $\underline{140}$

López, V. G., Marín, M. E., \& Alcalá, M. C. (2012). Ergonomía y productividad: Variables que se relacionan con la competitividad de las plantas maquiladoras. Revista de Ingeniería industrial: Actualidad y nuevas tendencias, $3(9), \quad 17-32$. http://servicio.bc.uc.edu.ve/ingenieria/revista/Inge-Industrial/volIII-ng/art2.pdf

Ministerio de la Protección Social (2007, 16 de agosto). Resolución No. 2844, por la cual se adoptan las Guías de Atención Integral de Salud Ocupacional Basadas en la Evidencia. Bogotá, Colombia. http://copaso.upbbga.edu.co/legislacion/resolucion 2844 colombia.pdf

Ministerio de Minas y Energía (2009, 6 de agosto). Resolución No. 18 1331. Continuación Anexo General Reglamento Técnico de Iluminación y Alumbrado Público - RETILAP. Bogotá, Colombia.

https://www.alcaldiabogota.gov.co/sisjurMantenimiento/adminverblobawa?tabla=T NOR MA ARCHIVO\&p NORMFIL ID=61\&f NORMFIL FILE=X\&inputfileext=NORMFIL FIL ENAME

Ministerio de Trabajo y Seguridad Social (1979, 22 de mayo). Resolución 240o, por la cual se 
establecen algunas disposiciones sobre vivienda, higiene $y$ seguridad en los establecimientos de trabajo. Bogotá, Colombia. https://www.ilo.org/dyn/travail/docs/1509/industrial\%20safety\%20statute.pdf

Ministerios de Trabajo y Seguridad Social y de Salud (1990, 3 de mayo). Resolución No. oo1792 por la cual por la cual se adoptan valores límites permisibles para la exposición ocupacional al ruido. Bogotá, Colombia. http://www.bogotaturismo.gov.co/sites/intranet.bogotaturismo.gov.co/files/RESOLUCI\%C 3\%93N\%201792\%20DE\%201990.pdf

Prokopenko, J. (1989). La gestión de la productividad. Manual Práctico ( $1^{\mathrm{a}}$ Ed). Oficina Internacional del Trabajo-OIT.

Rueda, M. J., \& Zambrano, M. (2013). Manual de Ergonomía y Seguridad (1 ${ }^{\text {a }}$ Ed). Alfaomega Colombiana.

Tafur, F. J. (2007). Informe de enfermedad profesional en Colombia, 2003-2005. Ministerio de la Protección Social.

Vallejo, J. (2000, septiembre). La ergonomía y su influencia en la productividad. Blog Ergonomía y Salud Ocupacional. http://ergounadantioquia.blogspot.com/p/articulos.html 

ANEXOS

Anexo 1. Ángulos corporales miembros superiores

\begin{tabular}{|c|l|}
\hline SEGMENTO & \multicolumn{1}{|c|}{ Movilidad articular } \\
\hline Hombro & $\begin{array}{l}\text { Flexión 180 grados, extensión 45 grados, rotación externa e } \\
\text { interna, 90grados, abducción y aducción, 180 grados. }\end{array}$ \\
\hline Codo & Flexión 145 grados, extensión 0 grados. \\
\hline Antebrazo & Supinación, 90 grados al igual que la pronación. \\
\hline Muñeca & $\begin{array}{l}\text { Inclinación Radial, 20 grados, inclinación Cubital, 45 grados, } \\
\text { flexión Dorsal, 70 grados, flexión Palmar, 80 grados. }\end{array}$ \\
\hline
\end{tabular}

Anexo 2. Lista de verificación de tornos.

\begin{tabular}{|c|c|c|c|c|}
\hline \multirow{2}{*}{ No } & \multirow{2}{*}{$\begin{array}{l}\text { ASPECTO A EVALUAR } \\
\text { SISTEMA ELÉCTRICO }\end{array}$} & \multicolumn{3}{|c|}{ ESTADO } \\
\hline & & Cumple & No cumple & No aplica \\
\hline 1 & $\begin{array}{l}\text { El interruptor de encendido funciona adecuadamente y está ubicado a } \\
\text { una distancia de fácil alcance o acceso }\end{array}$ & & & \\
\hline 2 & La carcaza metálica de la máquina está conectada a tierra & & & \\
\hline 3 & EL cable de alimentación está en buen estado (sin desgaste, œortes) & & & \\
\hline \multirow[t]{2}{*}{4} & $\begin{array}{l}\text { Los botones de parada de emergencia funcionan adecuadamente y } \\
\text { está ubicado a una distancia de fácil alcance o acceso }\end{array}$ & & & \\
\hline & NVELES DE ACEITE & & & \\
\hline 5 & Niveles de aceite caja de velocidades & & & \\
\hline 6 & Nivel de caja de avance & & & \\
\hline \multirow[t]{2}{*}{7} & Nivel de aceite en el soporte de árbol horizontal & & & \\
\hline & FUNCIONAMIENTO DE PARTES ELÉETRICAS & & & \\
\hline 8 & Làmpara de mesa longitudinal & & & \\
\hline 9 & Luces de tablero de encendido & & & \\
\hline 10 & Luces de tablero de control & & & \\
\hline 11 & Pulsadores de avance & & & \\
\hline 12 & Motor de caja de velocidades & & & \\
\hline \multirow[t]{2}{*}{13} & Motor de caja de avance & & & \\
\hline & COMPONENTES MECÁNICOS & & & \\
\hline 14 & Palanca de caja de velocidades & & & \\
\hline 15 & Palanca de caja de avance & & & \\
\hline 16 & Avance automático de cabeza horizontal & & & \\
\hline 17 & Sistema de bomba de refrigeración & & & \\
\hline \multirow[t]{2}{*}{18} & Ajuste adecuado de contrapunta y'o luneta & & & \\
\hline & HERRAMIENTAS DE CORTE & & & \\
\hline 19 & $\begin{array}{l}\text { Están en buen estado, con la geometria adecuada y con rompevirutas, } \\
\text { o con los parámetros adecuados }\end{array}$ & & & \\
\hline \multirow[t]{2}{*}{20} & Ubicadas o colocadas correctamente & & & \\
\hline & SEGURIDAD Y LIMPIEZA & & & \\
\hline 21 & $\begin{array}{l}\text { El torno v especialmente sus partes críticas y puntos de operación } \\
\text { (bancada, copa) se encuentran en buenas condiciones de limpieza }\end{array}$ & & & \\
\hline 22 & El piso es uniforme y no resbaloso & & & \\
\hline 23 & $\begin{array}{l}\text { La superficie del piso alrededor del torno se mantiene libre de aceite y } \\
\text { grasa }\end{array}$ & & & \\
\hline 24 & $\begin{array}{l}\text { Existen pantallas de resguardo contra riesgos de incendio para evitar la } \\
\text { proyección de virutas o astillas calientes }\end{array}$ & & & \\
\hline
\end{tabular}


Anexo 3. Descripción de las labores de los operarios de torno.

\begin{tabular}{|c|c|}
\hline \multicolumn{2}{|l|}{ TAREAS } \\
\hline DESCRIPCIÓN & $\begin{array}{c}\text { TIEMPO } \\
\text { ASIGNADO/JORNADA DIARIA }\end{array}$ \\
\hline $\begin{array}{l}\text { 1. Recepcionar y Analizar las especificaciones de los } \\
\text { requerimientos de la pieza o producto (Planos) y } \\
\text { devolverlo en caso de presentar alguna inconsistencia. }\end{array}$ & 5 minutos \\
\hline $\begin{array}{l}\text { 2. Revisar el funcionamiento del torno antes de operar y } \\
\text { luego aceitarlo. }\end{array}$ & 2 minutos \\
\hline 3. Alistar la herramienta a utilizar. & 3 minutos \\
\hline $\begin{array}{l}\text { 4. Operar el torno para la elaboración o reparación de piezas } \\
\text { de acuerdo con instrucciones, optimizando su } \\
\text { funcionamiento. }\end{array}$ & 420 minutos \\
\hline $\begin{array}{l}\text { 5. Refrigerar máquina y herramienta para evitar } \\
\text { recalentamiento (se acciona la llave de la bomba del agua, } \\
\text { previo encendido de la bomba con la mano derecha). }\end{array}$ & $\begin{array}{c}\text { De } 30 \text { segundos a } 1 \text { minuto } \\
\text { dependiendo el tamaño de la } \\
\text { pieza (la tarea se repite más del } \\
50 \% \text { de la jornada) }\end{array}$ \\
\hline $\begin{array}{l}\text { 6. Realizar seguimiento al trabajo realizado, verificando } \\
\text { calidad y medidas de acuerdo con plano (empleando } \\
\text { micrómetro y pie de rey). }\end{array}$ & $\begin{array}{c}\text { De } 5 \text { a } 30 \text { minutos } \\
\text { (dependiendo del tamaño de la } \\
\text { pieza) }\end{array}$ \\
\hline $\begin{array}{l}\text { 7. Asear el torno, la herramienta y el área de trabajo al } \\
\text { finalizar el turno. }\end{array}$ & 10 minutos \\
\hline 8. Ubicar la herramienta en su lugar. & 3 minutos \\
\hline
\end{tabular}


Anexo 4. Operaciones realizadas para la elaboración o reparación de piezas.

\begin{tabular}{|c|c|c|c|}
\hline \multicolumn{4}{|c|}{ OPERACION DE TORNO } \\
\hline No & OPERACIÓN & DESCRIPCIÓN & REGISTRO FOTOGRÁFICO \\
\hline 1 & $\begin{array}{l}\text { Posicionar la pieza en } \\
\text { la copa del torno, } \\
\text { apretando con la llave } \\
\text { de la copa }\end{array}$ & $\begin{array}{l}\text { Apretar con la llave de la } \\
\text { copa, empleando ambas } \\
\text { manos. }\end{array}$ & \\
\hline 2 & Hacer centro punto & $\begin{array}{l}\text { Empleando una broca centro } \\
\text { en el mandril se aprieta con } \\
\text { la mano derecha para darle } \\
\text { estabilidad a la pieza }\end{array}$ & \\
\hline 3 & $\begin{array}{l}\text { Posicionar la pieza en } \\
\text { el torno }\end{array}$ & $\begin{array}{l}\text { Ubicar la pieza en el torno } \\
\text { con el punto de la máquina } \\
\text { para que no genere } \\
\text { vibración, primero el seguro } \\
\text { más grande que se sujeta } \\
\text { con la bancada del torno y } \\
\text { luego se posiciona con el } \\
\text { seguro de punto }\end{array}$ & \\
\hline
\end{tabular}

\begin{tabular}{|l|l|l|}
\hline 4 & Procesar la pieza & $\begin{array}{l}\text { Con la mano izquierda se } \\
\text { alimenta para dar corte, se } \\
\text { gradúan las dimensiones } \\
\text { requeridas en milimetros con } \\
\text { la mano mecánica del torno } \\
\text { y se posiciona el automático. } \\
\text { Si se requiere disminuir el } \\
\text { tiempo de mecanizado se } \\
\text { devuelve el carro del torno } \\
\text { manualmente con la manija } \\
\text { del mismo, empleando la } \\
\text { mano derecha. }\end{array}$ \\
\hline 5 & Lijar la pieza & $\begin{array}{l}\text { Cuando se está terminando } \\
\text { la pieza se lija para pulir o } \\
\text { dar acabado y ajustarla a las } \\
\text { especificaciones del plano, } \\
\text { la operación puede tardar } \\
\text { entre } 1 \text { y } 5 \text { minutos } \\
\text { dependiendo del aumento } \\
\text { que se haya dejado para } \\
\text { darle pulimiento y del } \\
\text { tamaño de la pieza, se } \\
\text { realiza entre 10 y 20 veces } \\
\text { en el dia. } \\
\text { Cuando es una pieza } \\
\text { pequeña se sujeta la lija por } \\
\text { el extremo inferior con la } \\
\text { mano izquierda y con los } \\
\text { dedos pulgar e indice de la } \\
\text { mano derecha se desliza la } \\
\text { ija de izquierda a derecha } \\
\text { por la pieza. Cuando se trata }\end{array}$
\end{tabular}

\begin{tabular}{|c|c|c|c|}
\hline & & $\begin{array}{l}\text { de una pieza grande la } \\
\text { operación se realiza con } \\
\text { ambas manos (peso entre } 5 \\
\text { y } 10 \text { kilos). }\end{array}$ & \\
\hline 6 & Desmontar la pieza & $\begin{array}{l}\text { Se desajusta la copa del } \\
\text { torno con la llave } \\
\text { correspondiente, se } \\
\text { desmonta y se envia a } \\
\text { control de calidad para su } \\
\text { revisión y aprobación. Se } \\
\text { realiza al final del ciclo de } \\
\text { trabajo, puede tardar entre } \\
10 \text { y } 20 \text { segundos. }\end{array}$ & \\
\hline 7 & $\begin{array}{l}\text { Cambiar mordazas } \\
\text { para ubicar piezas } \\
\text { más grandes }\end{array}$ & $\begin{array}{l}\text { Se desajusta la copa del } \\
\text { tomo con la llave de copa, } \\
\text { se gradúan las mordazas y } \\
\text { se aplican las operaciones } \\
\text { descritas en los items } 1,2 \text { y } \\
\text { 3. }\end{array}$ & \\
\hline 8 & $\begin{array}{l}\text { Mover la posición de } \\
\text { una herramienta }\end{array}$ & $\begin{array}{l}\text { Se golpea con la mano la } \\
\text { torre del torno para que } \\
\text { ajuste en la nueva posición, } \\
\text { esta operación se realiza } \\
\text { una o dos veces diarias, en } \\
\text { ocasiones se golpea con un } \\
\text { bloque de plomo de } 1 \text { kilo } \\
\text { aproximadamente. }\end{array}$ & $\frac{2}{2 y}$ \\
\hline
\end{tabular}


Anexo 5. Entrada y salida de aire con equipo de ventilación instalado.

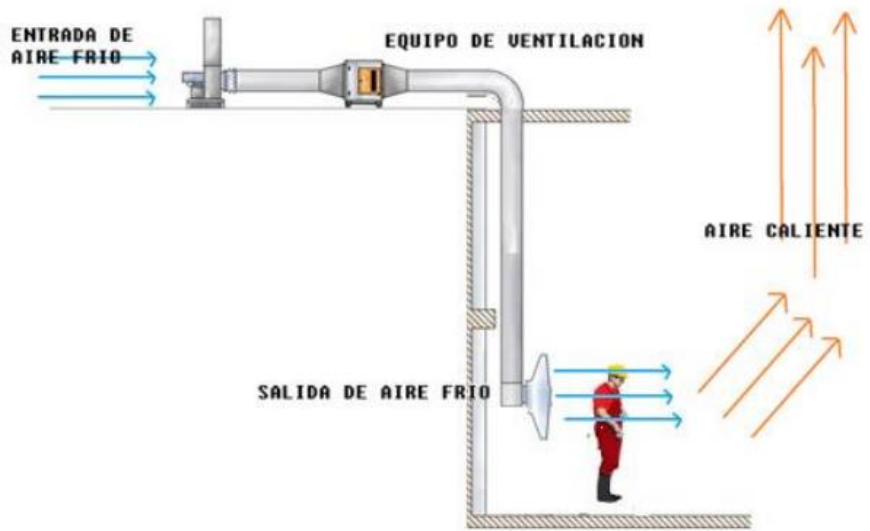

Anexo 6. Imágenes y especificaciones del equipo recomendado.
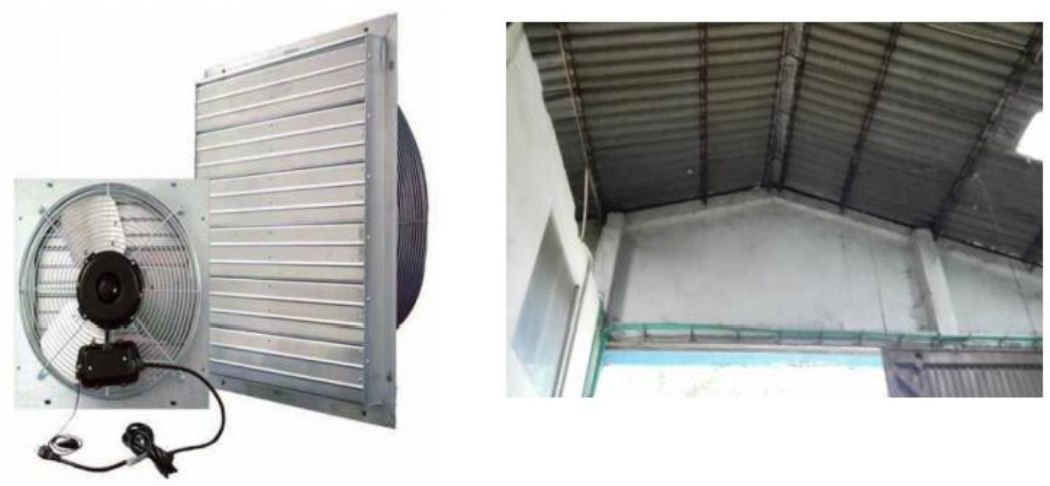

\begin{tabular}{|c|c|c|c|c|c|c|c|c|}
\hline Modelo & $\begin{array}{c}\text { Diámetro } \\
\text { de rotor } \\
\text { (In) }\end{array}$ & $\begin{array}{c}\text { Voltaje } \\
\text { (V) }\end{array}$ & $\begin{array}{c}\text { Motor } \\
\text { Monofásico } \\
\text { (HP) }\end{array}$ & $\begin{array}{c}\text { Nivel de } \\
\text { Ruido } \\
\text { (dBA)@3m }\end{array}$ & RPM & $\begin{array}{c}\text { Volumen } \\
\text { de Aire } \\
\text { (CFM) }\end{array}$ & $\begin{array}{c}\text { Peso } \\
\text { (Kg) }\end{array}$ & $\begin{array}{c}\text { Tamaño (L.W.H) } \\
\text { (mm) }\end{array}$ \\
\hline AirMAX 24-GL & 24 & 110 & $1 / 2(1 \mathrm{Ph})$ & 75 & 1750 & 6250 & 22 & $630 \times 630 \times 400$ \\
\hline
\end{tabular}

Anexo 7. Referencia ventilación por dilución.
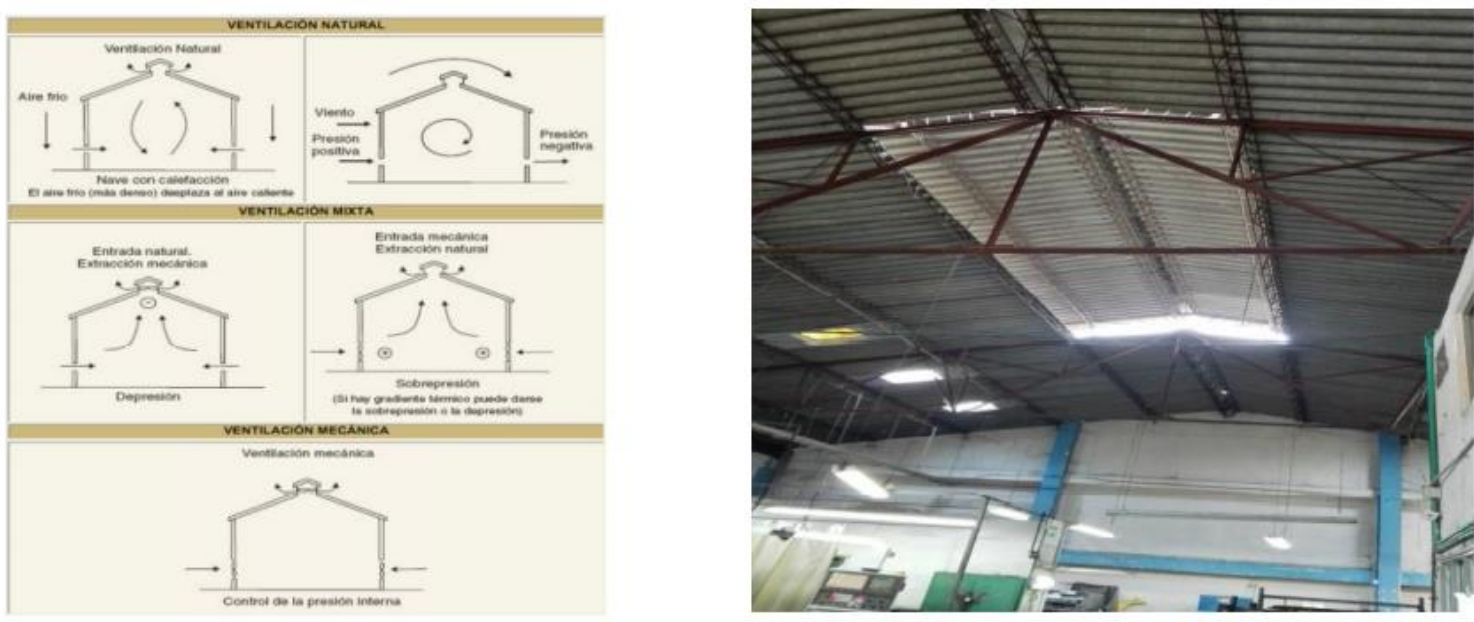